\title{
BUCHBESPRECHUNGEN
}

Böckstiegel:

Der Staat als Vertragspartner ausländischer

Privatunternehmer . . . . . . 373

Kebschull/Fasbender/Naini:

Entwicklungspolitik - eine Einführung . . 375

Musrey:

An Arab Common Market . . . . . . 376

\section{KarL-Heinz Böckstiegel}

Der Staat als Vertragspartner ausländischer Privatunternehmen

Völkerrecht und Internationales Wirtschaftsrecht, Bd. 1

Frankfurt/Main, Athenäum-Verlag

1971, 432 S., DM 58,-

Ohne sich mit historischen Reminiszenzen aufzuhalten, geht Böckstiegel sofort die systematisch-dogmatischen und praktischen Fragen der untersuchten Konzessions-, Investitionsverträge usw. an. Hier ist sogleich hervorzuheben, daß der Verfasser eine Unmenge an Material verarbeitet, das in dem stattlichen Literaturverzeichnis von 46 Seiten (leider fehlt allerdings die wichtige Haager Akademievorlesung von P. Weil mit demselben Titel: Problèmes relatifs aux contrats passés entre un Etat et un particulier, Recueils Bd. 128, 1969 III, S. 95-240) gar nicht in Erscheinung tritt, nämlich zahlreiche überhaupt nicht öffentlich einsehbare Konzessionsverträge usw., die dem Verfasser vertraulich von den Parteien selbst oder über die Internationale Handelskammer in Paris zugänglich gemacht wurden. Auch bei Respektierung dieses Vertrauens erreicht der Autor dadurch eine schwer wiederholbare Authentizität und Kompetenz in der Behandlung eines Problemkreises, der ganz wesentlich nicht nur zu einer Auflösung der Grenzen zwischen nationalem und internationalem, zwischen internationalem privaten und Völkerrecht (vgl. die jedoch im Ergebnis ablehnenden Ausführungen zur Theorie eines individuell-autonomen Vertragsrechts nach Verdross, S. 125 ff., und zu
Maxwell:

India's China War. . . 377

Gerold:

Die Sicherung des Friedens durch die

Organisation amerikanischer Staaten . . . 381

Moreno:

Barrios in Arms - Revolution in Santo

Domingo . . . . . 383

einer selbständigen Rechtsmasse "transnationales Wirtschaftsrecht", S. 136 ff.), sondern darüber hinaus zur Infragestellung der Kategorien „national“ und „international" überhaupt und dem entsprechenden Versuch geführt hat, sie durch neue Begriffe transnationaler Interaktionensysteme o. ä. abzulösen 1 .

Das Buch zerfällt in drei Teile. Der erste ist der Frage gewidmet, wer im Rahmen der untersuchten Verträge als staatlicher Partner anzusehen ist, denn häufig tritt der Staat nicht unmittelbar selbst als Teilnehmer am internationalen Wirtschaftsverkehr auf. Hier macht die Identifizierung, anders als auf seiten des privaten Partners, oft erhebliche Schwierigkeiten. Böckstiegel vermeidet generalisierende Definitionen und stellt auf die im Zusammenhang des konkreten Vertrages und im Hinblick auf die konkret $\mathrm{zu}$ entscheidende jeweilige Rechtsfrage vorzunehmende Identifikation der "funktionellen" Staatlichkeit ab. Er entwickelt eine Reihe von Kriterien, nach denen im Recht der BRD und vor allem im internationalen Recht der „funktionale Durchgriff“ auf den hinter einer selbständig auftretenden Rechtspersönlichkeit stehenden Staat für möglich erachtet wird. Infolge der Gewichtsverteilung in dem Buche wird diesen Ausführungen durch das in Teil III behandelte Thema der beschränkten Völkerrechtsqualität dieser Verträge, das offenbar das Hauptinteresse Böckstiegels findet, in gewisser Weise der Boden entzogen. Voraussetzung dieser Qualität nämlich ist nach Ansicht des Verfassers (u. a.), daß die Verträge von den zum Abschluß völkerrechtlicher

1 Vgl. K. Kaiser, Transnationale Politik. Zu einer Theorie der multinationalen Politik, Politische Vierteljahresschrift, Sonderheft 1 (1969), S. $80 \mathrm{ff.}$ 
Verträge befugten höchsten Staatsstellen abgeschlossen oder genehmigt werden (S. 205 f., 310). Damit aber ist wohl zugleich die Frage nach der funktionellen Staatlichkeit mit beantwortet - der Staat handelt hier unmittelbar. Aber es gibt natürlich auch andere Verträge.

In Teil II („Vertrag und Rechtsordnung") behandelt Böckstiegel die denkbaren Beziehungen zwischen den untersuchten Verträgen und den in Frage kommenden Rechtsordnungen („Zuordnung" als Oberbegriff). Besonders hervorzuheben ist hier die Herausarbeitung der unterschiedlichen Ebenen der Zuordnung. Generell wie für den Fortgang der Arbeit besonders wichtig ist die präzise Unterscheidung $z$ wischen der Ebene, in der die Verpflichtungswirkung des Vertrages wurzelt, und jener, die die Herrschaft über den Inhalt der Vertragspflichten, das "proper law" des Vertrages, konstituiert. Der Verfasser untersucht, wiederum an Hand einer reichen Praxis, die Rechtswahlmöglichkeiten für diese verschiedenen Ebenen innerhalb der Vertragsautonomie, also auch die Wahl z. B. des Völkerrechts, ferner die Grenzen dieser Autonomie im nationalen und im Völkerrecht, die schon erwähnte Möglichkeit einer neuen besonderen Rechtsmasse usw.

In dem fast zwei Drittel des Buches umfassenden Teil III schließlich geht es darum, ob durch eine „Vervölkerrechtlichung " dieser Verträge den privaten Vertragspartnern größere Sicherheit, damit Vertrauen und Unternehmenswie Investitionsbereitschaft gegeben werde, an denen ja besonders die jungen Staaten in erster Linie selbst interessiert seien - allen Vorwürfen des Neokolonialismus usw. zum Trotz -, was sie ja durch den Abschluß einschlägiger Verträge immer wieder dokumentierten. Dieses Problemverständnis der privaten Investitions- und Unternehmertätigkeit in den Entwicklungsländern grenzt an Naivität. - Mit der
Böckstiegel genügenden Konstatierung der Tatsache des Abschlusses derartiger Verträge setzt das Fragen nach der Problematik dieser Zusammenhänge ja erst ein, etwa im Hinblick auf das verstärkte Interesse der USA an solchen Privatinvestitionen ${ }^{2}$. Hier kann nur beispielhaft auf die sehr abgewogene Darstellung dieser Problematik bei R. Kapfe$\mathrm{rer}^{3}$ oder die kritischere bei E. Krippendorff ${ }^{4}$ verwiesen werden.

Wichtig für diese völkerrechtliche Absicherung, die angesichts der häufigen Vereinbarung von Schiedsklauseln sogar eine Chance der Durchsetzbarkeit erhält, muß die Wahl des Völkerrechts nicht nur als "proper law", sondern als Quelle der Verpflichtungswirkung des Vertrages sein, denn nur dann wird die Vertragspflicht $\mathrm{zu}$ einer echten völkerrechtlichen, für die der Grundsatz pacta sunt servanda unmittelbar gilt; dann nämlich genießt die ausländische Investition nicht nur Wertbestandsschutz durch die völkerrechtlichen Eigentumsschutznormen - Enteignung ist gegen Entschädigung zulässig (mit den einschlägigen Problemen auf S. 164 ff. erörtert) - , sondern Enteignung wird dann völkerrechtlich unzulässig, ihre Vornahme ist direkter Völkerrechtsbruch mit allen daraus sich ergebenden Folgen. Als Mittel für diesen Zweck kommen einmal echte Staatsverträge mit dem Heimatstaat des ausländischen Investors usw. in Betracht (S. 146 ff.), die die Garantien der - heute - Entwicklungsländer zugunsten der privaten Vertragspartner in völkerrechtliche Verpflichtungen gegenüber deren Heimatstaat transformieren. Verletzungen der (privat-)vertraglichen Rechte des Investors werden dadurch zur Völkerrechtsverletzung gegenüber dessen Heimatstaat. Die einschlägigen früheren bilateralen Handels- und Niederlassungs-, heute Investitionsschutzverträge usw. sowie multilaterale Ansätze werden untersucht.

2 Vgl. dazu Präsident Nixons Auslandshilfe-Botschaften an den Kongreß vom 15. 9. 1970, Dpt. St. Bull. Bd. 63 (1970) II S. 369, und vom 21. 4.1971 , ibid. Bd. 64 (1971) I, S. 614, und die daraufhin gegründete Overseas Private Investment Corporation.

3 Entwicklungshilfe zwischen Neo-Imperialismus und Weltinnenpolitik. Eine Bilanz am Ende der ersten Entwicklungsdekade. Aus Politik und Zeitgeschichte, Beilage 24/71 zu "Das Parlament ${ }^{\alpha}$ v. 12. 6. 1971.

4 Die Amerikanische Strategie (Frankfurt/Main 1970), bes. S. $370 \mathrm{ff}$. 
Der interessanteste Ansatz liegt jedoch in der Konzeption der beschränkt völkerrechtlichen Verträge (S. 177 ff.), die Böckstiegel unter bestimmten Voraussetzungen (die wichtigste - Abschluß durch höchste Staatsstellen - wurde schon erwähnt, vgl. im übrigen die $\mathrm{Zu}$ sammenfassung S. 310) für möglich hält. Dabei leitet er die auf den Funktionszusammenhang des Vertrages (ähnlich wie bei internationalen Organisationen auf Regierungsebene) "beschränkte“ Völkerrechtssubjektivität des privaten Vertragspartners von seinem staatlichen Partner ab. Das kann er tun, indem er die (von W. Rudolf stammende) Definition des Völkerrechts zugrunde legt, nach welcher seine Subjekte die zur internationalen Staatengemeinschaft gehörenden Staaten und die von diesen als gleichberechtigt Anerkannten sind (S. 182). - $\mathrm{Zu}$ fragen wäre indessen, ob man angesichts der Bedeutung dieses Problems nicht zu einem breiteren theoretischen Fundament gelangen könnte. - Böckstiegel diskutiert des weiteren völkergewohnheitsrechtliche Tendenzen, die seine Thesen stützen, aber auch Grenzen der völkerrechtlichen Bindung, die sich aus dem Wesen des Vertrages, der Natur der Staatlichkeit des einen und der Nichtstaatlichkeit des anderen Partners ergeben - Überlegungen und Informationen, die das Buch geradezu faszinierend und auch für den mit diesen Fragen befaßten Praktiker fruchtbar machen. - Man hätte sich allerdings sorgfältigere Korrekturen gewünscht. Fängt man erst an, darauf zu achten, finden sich allzu viele Druckfehler: z. B. "der" statt "den“ auf S. 249; "Rechtsmaße“ statt "Rechtsmasse“ auf S. 250; seitenvertauschte Anmerkungen, Nr. 725, 726 auf S. 329/330 usw.

Knud Krakau
Dietrich Kebschull

unter Mitarbeit von

Karl Fasbender und Ahmad Naini

Entwicklungspolitik - Eine Einführung

Veröffentlichung des HWWA-Institut

für Wirtschaftsforschung - Hamburg

Bertelsmann Universitätsverlag, Düsseldorf 1971, 192 Seiten, DM 19,80.

Die lobenswerte Absicht des Verfassers und seiner beiden Mitarbeiter „allgemeinverständlich in das Gebiet der Entwicklungspolitik einzuführen", ist mit dem vorliegenden Buch recht gut verwirklicht worden. Allerdings - was hier als Einführung angekündigt wird, entpuppt sich - zumindest im ökonomischen Bereich - als weit mehr: Es werden nahezu alle wirtschaftlichen Aspekte der Entwicklungspolitik beleuchtet. Zunächst bietet das Buch eine ausführliche Analyse der Situation in den Entwicklungsländern. Sie umfaßt das Bevölkerungsproblem, die Lage in den drei Hauptsektoren der Wirtschaft sowie die Außenwirtschaft, die Infrastruktur und das Bildungswesen. Eine Untersuchung über die Motive und die allgemeinen Zielsetzungen der Entwicklungspolitik der Industrieländer schließt sich an. Hierbei wird geprüft, inwieweit sich deren entwicklungspolitische Zielvorstellungen mit den Erfordernissen der Entwicklungsländer decken.

Einen breiten Raum nimmt die Erörterung der unterschiedlichen Formen der Entwicklungshilfe ein. Diese Analyse wird konkretisiert durch Angaben über die Hilfsleistungen einzelner Staaten, insbesondere der Bundesrepublik Deutschland. Der weitgehend deskriptive Teil über deutsche und internationale Organisationen der Entwicklungshilfe erscheint in diesem Rahmen $\mathrm{zu}$ lang geraten. Dadurch wird der eigentliche Schwerpunkt dieses $\mathrm{Bu}$ ches - die Frage nach der optimalen Strategie der Entwicklungspolitik hinten angesetzt. Der Verfasser kommt hier zu dem Ergebnis, daß weder eine Marktwirtschaft westlicher Prägung noch eine Zentralverwaltungswirtschaft nach östlichem Muster für die Entwicklungsländer der geeignete Weg seien. 